\title{
Designing the Search Experience
}

\author{
Tony Russell-Rose \\ UXLabs Ltd. and City University London \\ Northampton Square, London EC1V 0HB \\ tgr@uxlabs.co.uk
}

\begin{abstract}
This half-day tutorial provides a practical introduction to HumanCentred Design for information search, access and discovery. We present a concise overview of the fundamental concepts and principles of human information-seeking behaviour and show how to apply these in the design of search user experiences. A key element of the tutorial is the opportunity to practice these skills in a group exercise.
\end{abstract}

Keywords: search, navigation, information retrieval, information discovery, data visualization, user experience, user-centred design.

\section{Tutorial Goals}

Participants in this half-day tutorial will:

- Explore the fundamental concepts and principles of Human-Centred Design for information search, access and discovery;

- Learn how to differentiate between various types of search behaviour: known-item, exploratory, etc.;

- Study models of human information-seeking behaviour (e.g. Broder, Norman, Marchionini, Bates, etc.), and how to apply interaction design principles based on those models;

- Develop an understanding of the key dimensions of user type, goal and mode of interaction, and how to apply these variables when designing for different user contexts;

- Understand the role of design patterns, and how to apply UI design patterns from various libraries in designing search user interfaces;

- Gain an awareness of the key design resources available within the HCIR community and how to apply these to practical design challenges.

\section{Content}

1. Introductions and objectives: Group introductions \& Ice-breaker. A brief summary of what each participant hopes to gain from the session, and what experiences they bring. 
2. Understanding Search \& Discovery Behaviour: An overview of the key models and frameworks for human-information seeking, focusing on the work of Marchionini, Bates, and more contemporary works of Morville, Tunkelang at al.

3. Faceted Classification \& Search: A review of Ranganathan's seminal work on Colon Classification and an exploration the implications for the design of contemporary faceted classification and search paradigms.

4. Varied Solutions for Varied Contexts: An exploration of the universal dimensions that define search and discovery experiences, and how these translate into principles for the design of contemporary faceted search experiences.

5. UI Design Pattern Libraries: A detailed examination of best practices in search experience design, embodied as design patterns in key HCIR design pattern libraries.

6. Exercise: UX Review: An opportunity to practice all the above skills in a group exercise. The scenario will involve critical review of a live site, with analysis and review of the search and discovery experience, and synthesis of appropriate design insight and recommendations.

7. Exercise: UX Review (Feedback \& Presentations): Feedback and review of the group exercise.

8. Conclusions \& Wrap-up: A review of the overall session, including the shared experiences of the group exercises and the contrasting findings of each.

\section{Intended Audience}

This tutorial is aimed at information architects, user experience architects, search specialists, and HCI practitioners and researchers interested in the designing more effective user experiences for search and information discovery.

\section{Presenter's Background}

Tony Russell-Rose is currently director of UXLabs, a consultancy specialising in user experience research, design and analytics. Before founding UXLabs he was Manager of User Experience at Endeca and editor of the Endeca UI Design Pattern Library, an online resource dedicated to best practice in the design of search and discovery experiences.

Prior to this he was technical lead at Reuters, specialising in advanced user interfaces for information access and search. And before Reuters he was R\&D group manager at Canon Research Centre Europe, where he led a team developing next generation information access products and services. Earlier professional experience includes a Royal Academy of Engineering fellowship at HP Labs and a Short-term Research Fellowship at BT Labs. His academic qualifications include a PhD in human-computer interaction, an MSc in cognitive psychology and a first degree in engineering, majoring in human factors. Tony is also Honorary Visiting Fellow at the Centre for Interactive Systems Research, City University London. 